\title{
Some results on the main supergraph of finite groups
}

\author{
A. K. Asboei and S. S. Salehi \\ Communicated by A. Yu. Olshanskii
}

\begin{abstract}
A BSTRACT. Let $G$ be a finite group. The main supergraph $\mathcal{S}(G)$ is a graph with vertex set $G$ in which two vertices $x$ and $y$ are adjacent if and only if $o(x) \mid o(y)$ or $o(y) \mid o(x)$. In this paper, we will show that $G \cong \operatorname{PSL}(2, p)$ or $\operatorname{PGL}(2, p)$ if and only if $\mathcal{S}(G) \cong \mathcal{S}(\operatorname{PSL}(2, p))$ or $\mathcal{S}(\operatorname{PGL}(2, p))$, respectively. Also, we will show that if $M$ is a sporadic simple group, then $G \cong M$ if only if $\mathcal{S}(G) \cong \mathcal{S}(M)$.
\end{abstract}

\section{Introduction}

Let $G$ be a finite group and $x \in G$. The order of $x$ is denoted by $o(x)$. The set of all element orders of $G$ is denoted by $\pi_{e}(G)$ and the set of all prime divisors of $|G|$ is denoted by $\pi(G)$. It is clear that the set $\pi_{e}(G)$ is closed and partially ordered by divisibility, and hence it is uniquely determined by $\mu(G)$, the subset of its maximal elements. Set $m_{i}=m_{i}(G)=|\{g \in G \mid o(g)=i\}|$. In this paper, we use $p$ for denoting a prime number unless specifically stated otherwise.

We define the graph $\mathcal{S}(G)$ with vertex set $G$ such that two vertices $x$ and $y$ are adjacent if and only if $o(x) \mid o(y)$ or $o(y) \mid o(x)$. This graph is called the main supergraph of power graph $G$ and was introduced in [13]. Power graph $\mathcal{P}(G)$ is a graph with the vertex set $G$, in which two distinct elements are adjacent if one is a power of the other. The main properties

2010 MSC: 20D08; 05C25.

Key words and phrases: graph, main supergraph, finite groups, Thompson's problem. 
of this graph were investigated by Cameron [9] and Chakrabarty et al. [10]. The proper main supergraph $\mathcal{S}^{*}(G)$ are defined as graphs constructed from $\mathcal{S}(G)$ by removing the identity element of $G$. We write $x \sim y$ when two vertices $x$ and $y$ are adjacent.

We say that groups $G_{1}$ and $G_{2}$ are of the same order type if and only if $m_{t}\left(G_{1}\right)=m_{t}\left(G_{2}\right)$ for all $t$. By the definition of the main supergraph, it is clear that if $G_{1}$ and $G_{2}$ are groups with the same order type, then $\mathcal{S}\left(G_{1}\right) \cong \mathcal{S}\left(G_{2}\right)$. The converse of this result is not generally correct. To prove, we consider $G_{1}=Z_{4} \times Z_{4}$ and $G_{2}=Z_{4} \times Z_{2} \times Z_{2}$. Since $G_{1}$ and $G_{2}$ are 2 -groups, we have $\mathcal{S}\left(G_{1}\right) \cong \mathcal{S}\left(G_{2}\right)$. But $m_{4}\left(G_{1}\right)=12>8=m_{4}\left(G_{2}\right)$ and $m_{2}\left(G_{1}\right)=3<7=m_{2}\left(G_{2}\right)$.

In 1987, J. G. Thompson [16, Problem 12.37] posed the following problem:

Thompson's Problem. Suppose that $G_{1}$ and $G_{2}$ are two groups of the same order type. If $G_{1}$ is solvable, is it true that $G_{2}$ is also necessarily solvable?

Let nse $(G)$ be the set of the number of elements of the same order in $G$. If $G_{1}$ and $G_{2}$ are the same order type, then $\operatorname{nse}\left(G_{1}\right)=\operatorname{nse}\left(G_{2}\right)$ and $\left|G_{1}\right|=\left|G_{2}\right|$. Therefore, if a group $G$ has been uniquely determined by its order and nse $(G)$, then Thompson's problem is true for $G$. In $[1,4,6]$, it is proved that the group $\operatorname{PSL}(2, p), \operatorname{PGL}(2, p)$, and the sporadic simple groups characterizable by their nse and order. Consequently, there is no solvable group that has the same order type as $\operatorname{PSL}(2, p), \operatorname{PGL}(2, p)$, and the sporadic simple groups.

Clearly, for two groups $G_{1}$ and $G_{2}$ that are the same order type, $\mathcal{S}\left(G_{1}\right) \cong \mathcal{S}\left(G_{2}\right)$. So, if a group $G$ has been uniquely determined by $\mathcal{S}(G)$, then Thompson's problem is true for $G$. In $[5,7,8]$, the authors of this paper proved that the alternating group of degree $p, p+1, p+2$, the symmetric group of degree $p$, the small Ree group ${ }^{2} G_{2}\left(3^{2 n+1}\right)$, and the Suzuki group are uniquely determined by their main supergraph. So, there is no solvable group that has the same order type as these simple groups. Also, in this paper, by the main supergraph, we show that there is no solvable group that has the same order type as $\operatorname{PSL}(2, p), \operatorname{PGL}(2, p)$, and the sporadic simple groups.

We construct the prime graph of $G$, which is denoted by $\Gamma(G)$, as follows: the vertex set is $\pi(G)$ and two distinct vertices $p$ and $q$ are joined by an edge if and only if $G$ has an element of order $p q(p \neq q)$. Let $t(G)$ be the number of connected components of $\Gamma(G)$ and let $\pi_{1}, \pi_{2}, \ldots, \pi_{t(G)}$ be the connected components of $\Gamma(G)$. If $2 \in \pi(G)$, then we always suppose 
$2 \in \pi_{1}$. If $r \in \pi(G)$ and $\{r\}$ is a connected component of $\Gamma(G)$, then we say $r$ is an isolated vertex of the prime graph of $G$.

Throughout this paper we denote by $\phi$ the Euler's totient function. We denote by $G_{q}$ a Sylow $q$-subgroup of $G$ and $n_{q}(G)$ is the number of Sylow $q$-subgroup of $G$, that is, $n_{q}(G)=\left|\operatorname{Syl}_{q}(G)\right|$. The other notation and terminology in this paper are standard, and the reader is referred to [11] if necessary.

\section{Preliminary results}

In this section, we present some preliminary results which will turn out to be useful in what follows. We start with a classical theorem of Frobenius.

Lemma 1 ([12]). Let $G$ be a finite group and $m$ be a positive integer dividing $|G|$. If $L_{m}(G)=\left\{g \in G \mid g^{m}=1\right\}$, then $m|| L_{m}(G) \mid$.

Lemma 2 ([2, Theorem 2.3]). Let $p$ be a prime that is not a Mersenne prime. If $G$ is a group satisfying

(1) $|G|=|\operatorname{PSL}(2, p)|$,

(2) $\left|\operatorname{Syl}_{p}(G)\right|=\left|\operatorname{Syl}_{p}(\operatorname{PSL}(2, p))\right|$, then $G \cong \operatorname{PSL}(2, p)$.

Lemma 3 ([2, Theorem 2.5]). Let $p$ be a Mersenne prime. If $G$ is a group satisfying

(1) $|G|=|\operatorname{PSL}(2, p)|$,

(2) $\left|\operatorname{Syl}_{p}(G)\right|=\left|\operatorname{Syl}_{p}(\operatorname{PSL}(2, p))\right|$, and

(3) $p$ is an isolated vertex of the prime graph of $G$, then $G \cong \operatorname{PSL}(2, p)$ unless it is the case that $p=7$ and $G \cong \mathrm{P \Gamma L}_{2}(8)$.

Lemma 4 ([3, Theorem 1.1]). Let $p$ be a prime and $G$ be a group such that $|G|=|\operatorname{PGL}(2, p)|$ and $\left|N_{G}(R)\right|=\left|N_{\mathrm{PGL}(2, p)}(S)\right|$, where $R \in \operatorname{Syl}_{p}(G)$ and $S \in \operatorname{Syl}_{p}(\mathrm{PGL}(2, p))$. Then the following assertions are true.

(1) If $p$ is not a Mersenne prime, then $G$ is isomorphic to $\operatorname{PSL}(2, p) \times C_{2}$, $\mathrm{SL}(2, p)$ or $\mathrm{PGL}(2, p)$.

(2) If $p>3$ is a Mersenne prime and $p$ is an isolated vertex of the prime graph of $G$, then $G \cong \operatorname{PGL}(2, p)$.

Lemma 5 ([15]). Let $S$ be a sporadic simple group and $p$ be the greatest element of $\pi(S)$. Then $S$ is uniquely determined by $|S|$ and $n_{p}(S)$. 
Remark 1. Let $m_{n}=m_{n}(G)$ be the number of elements of order $n$ of a finite group $G$. We note that $m_{n}=k \phi(n)$, where $k$ is the number of cyclic subgroups of order $n$ in $G$. If $n|| G \mid$, then by Lemma 1 we have $\phi(n) \mid m_{n}$ and $n \mid \sum_{d \mid n} m_{d}$.

\section{Main results}

In this section, we prove that the groups $\operatorname{PSL}(2, p), \operatorname{PGL}(2, p)$, and the sporadic simple groups uniquely determined by their main supergraph.

Theorem 1. Let $G$ be a finite group and $p>2$ a prime number. If $\mathcal{S}(\operatorname{PSL}(2, p)) \cong \mathcal{S}(G)$, then $G \cong \operatorname{PSL}(2, p)$.

Proof. By the definition of the main supergraph and our assumption, we have $|G|=|\operatorname{PSL}(2, p)|$. Also, by $\mathcal{S}(\operatorname{PSL}(2, p)) \cong \mathcal{S}(G)$ and the definition of the proper main supergraph, we have $\mathcal{S}^{*}(\operatorname{PSL}(2, p)) \cong \mathcal{S}^{*}(G)$.

By $[14$, p. 213], $\mu(\operatorname{PSL}(2, p))=\{(p-1) / 2, p,(p+1) / 2\}$. Thus $\operatorname{PSL}(2, p)$ has not any element of order $r p$, where $r \in \pi(G)$. By [1, Lemmas 2.3 and 2.4], $\operatorname{PSL}(2, p)$ contains exactly $p^{2}-1$ elements of order $p$. It follows that $\mathcal{S}^{*}(G)$ is a disconnected graph and at least one of the connected components of this graph is a complete graph of order $p^{2}-1$. Denote by $K_{p^{2}-1}$ a complete subgraph of order $p^{2}-1$ in the graph $\mathcal{S}^{*}(G)$. We prove that the vertices of $K_{p^{2}-1}$ are elements of order $p$.

First, let $x$ and $y$ be two vertices of $K_{p^{2}-1}$ such that $o(x)=r, o(y)=s$, $r, s \in \pi(G)$ and $r \neq s$. Since $K_{p^{2}-1}$ is a complete graph, we have $x \sim y$, which is a contradiction. Let $r$ be a prime and the vertices of $K_{p^{2}-1}$ be all of $x \in G$ such that $o(x)=r, r^{2}, \ldots$, or $r^{k}$ (note that $\exp \left(G_{r}\right)=r^{k}$ ). Then with considering $n=\left|G_{r}\right|$ in Remark 2.1, $\left|G_{r}\right| \mid\left(1+m_{r}+m_{r^{2}}+\ldots+m_{r^{k}}\right)=$ $1+p^{2}-1=p^{2}$. It follows that $r=p$. Hence, the vertices of $K_{p^{2}-1}$ are $x \in G$ such that $o(x)=p^{k}$, where $k \geqslant 1$ is an integer.

Since $|G|=p\left(p^{2}-1\right) / 2$, we have $p^{2} \nmid|G|$ and so $p^{2} \notin \pi_{e}(G)$. Therefore, the vertices of $K_{p^{2}-1}$ is all of elements of order $p$ in $G$. Thus $m_{p}(G)=p^{2}-1$.

Let $p r \in \pi_{e}(G)$, where $r \in \pi(G) \backslash\{p\}$. Then there exists an element $x \in G$ such that $o(x)=r$ and $x$ is one of the vertices of $K_{p^{2}-1}$, which is a contradiction. Thus $p$ is an isolated vertex of the prime graph of $G$.

By Remark 1, $m_{p}(G)=\phi(p) n_{p}(G)=(p-1) n_{p}(G)=p^{2}-1$, so $n_{p}(G)=p+1$. By Lemmas 2 and $3, G \cong \operatorname{PSL}(2, p)$ unless it is the case that $p=7$. If $p=7$, then $G \cong \operatorname{PSL}(2,7)$ or $\mathrm{P}_{2}(8)$. Let $G \cong \mathrm{P}_{2}(8)$. We have $\pi_{e}\left(\mathrm{P} \Gamma L_{2}(8)\right)=\{1,2,3,6,7\}$. So, $\mathcal{S}^{*}\left(\mathrm{P} L_{2}(8)\right)$ has two connected components, but $\mathcal{S}^{*}(\operatorname{PSL}(2,7))$ has three connected components, we get 
a contradiction by $\mathcal{S}^{*}(\operatorname{PSL}(2,7)) \cong \mathcal{S}^{*}(G)$. Therefore, $G \cong \operatorname{PSL}(2,7)$ for the case $p=7$. The proof is completed.

Theorem 2. Let $G$ be a finite group and $p>2$ a prime number. If $\mathcal{S}(\operatorname{PGL}(2, p)) \cong \mathcal{S}(G)$, then $G \cong \operatorname{PGL}(2, p)$.

Proof. The proof of this theorem is similar to the proof of the Theorem 1. Since some part of the proof is different from Theorem 1, we have repeated the proof in the following.

By the definition of the main supergraph and our assumption, we have $|G|=|\operatorname{PGL}(2, p)|$ and $\mathcal{S}^{*}(\operatorname{PGL}(2, p)) \cong \mathcal{S}^{*}(G)$.

By $[17$, Lemma 2.1], $\mu(\operatorname{PGL}(2, p))=\{p-1, p, p+1\}$. Thus PGL $(2, p)$ has not any element $r p$, where $r \in \pi(G)$. By [4, Lemma 2.4], $G$ contains exactly $p^{2}-1$ elements of order $p$. It follows that $\mathcal{S}^{*}(G)$ is a disconnected graph and at least one of the connected components of this graph is a complete graph of order $p^{2}-1$. Denote by $K_{p^{2}-1}$ a complete subgraph of order $p^{2}-1$ in the graph $\mathcal{S}^{*}(G)$. We prove that the vertices of $K_{p^{2}-1}$ are elements of order $p$.

First, let $x$ and $y$ be two vertices of $K_{p^{2}-1}$ such that $o(x)=r, o(y)=s$, $r, s \in \pi(G)$ and $r \neq s$. Since $K_{p^{2}-1}$ is a complete graph, we have $x \sim y$, which is a contradiction. Let $r$ be a prime and the vertices of $K_{p^{2}-1}$ be $x \in G$ such that $o(x)=r, r^{2}, \ldots, r^{k}$ (note that $\left.\exp \left(G_{r}\right)=r^{k}\right)$. Then with considering $n=\left|G_{r}\right|$ in Remark $1,\left|G_{r}\right| \mid\left(1+m_{r}+m_{r^{2}}+\ldots+m_{r^{k}}\right)=$ $1+p^{2}-1=p^{2}$. It follows that $r=p$. Hence, the vertices of $K_{p^{2}-1}$ are $x \in G$ such that $o(x)=p^{k}$, where $k \geqslant 1$ is an integer.

Since $|G|=p\left(p^{2}-1\right)$, we have $p^{2} \nmid|G|$ and so $p^{2} \notin \pi_{e}(G)$. Therefore, the vertices of $K_{p^{2}-1}$ is all of elements of order $p$ in $G$. Thus $m_{p}(G)=p^{2}-1$.

Let $p r \in \pi_{e}(G)$, where $r \in \pi(G) \backslash\{p\}$. Then there exists an element $x$ in $G$ such that $o(x)=r$ and $x$ is one of the vertices of $K_{p^{2}-1}$, a contradiction.

By Remark 1, $m_{p}(G)=\phi(p) n_{p}(G)=(p-1) n_{p}(G)=p^{2}-1$, so $n_{p}(G)=p+1$.

If $p$ is not a Mersenne prime, then by Lemma $4 G$ is isomorphic to $\operatorname{PSL}(2, p) \times C_{2}, \operatorname{SL}(2, p)$ or $\operatorname{PGL}(2, p)$. Let $G$ be isomorphic to $\operatorname{PSL}(2, p) \times$ $C_{2}$ or $\mathrm{SL}(2, p)$. Then $G$ has an element of order $2 p$, which is a contradiction. Therefore, $G \cong \operatorname{PGL}(2, p)$.

If $p$ is a Mersenne prime, then by Lemma $4, G \cong \operatorname{PGL}(2, p)$.

Theorem 3. Let $G$ be a finite group. If $\mathcal{S}(M) \cong \mathcal{S}(G)$ where $M$ is a sporadic simple group, then $G \cong M$.

Proof. Since $\mathcal{S}(M) \cong \mathcal{S}(G)$, we have $|G|=|M|$ and $\mathcal{S}^{*}(M) \cong \mathcal{S}^{*}(G)$. Let $p$ be the greatest element of $\pi(M)$. By [11], $p^{2} \nmid|G|$ and $p r \notin \pi_{e}(M)$ for 
every $r \in \pi(M)$. Thus $\mathcal{S}^{*}(M) \cong \mathcal{S}^{*}(G)$ is a disconnected graph and at least one of the connected components of this graph is complete graph of order $t=m_{p}(M)$. Denote by $K_{t}$ a complete subgraph of order $t$ in the graph $\mathcal{S}^{*}(G)$. We will show that the vertices of $K_{t}$ are elements of order $p$.

First, let $x$ and $y$ be two vertices of $K_{t}$ such that $o(x)=r, o(y)=s$, $r, s \in \pi(G)$ and $r \neq s$. Since $K_{t}$ is a complete graph, we have $x \sim y$, a contradiction. Let $r$ be a prime and the vertices of $K_{t}$ be all of $x \in G$ such that $o(x)=r, r^{2}, \ldots$, or $r^{k}$ (note that $\left.\exp \left(G_{r}\right)=r^{k}\right)$. Then by Remark 1 , $\left|G_{r}\right| \mid\left(1+m_{r}+m_{r^{2}}+\ldots+m_{r^{k}}\right)=1+t$. Now, it is easy to check (case by case for all of sporadic simple group $M$ ) that $r=p$. Hence, the vertices of $K_{t}$ are $x \in G$ such that $o(x)=p^{k}$, where $k \geqslant 1$ is an integer.

Since $p^{2} \nmid|G|$, we have $p^{2} \notin \pi_{e}(G)$. Therefore, the vertices of $K_{t}$ is all of elements of order $p$ in $G$. Hence, $m_{p}(G)=m_{p}(M)$. Remark 1 follows that $G$ and $M$ have equal numbers of Sylow $p$-subgroups and by Lemma 5 , $G$ is isomorphic to $M$ and now the proof is completed.

By our example in the introduction, we have seen if $\mathcal{S}\left(G_{1}\right) \cong \mathcal{S}\left(G_{2}\right)$, then it is not necessary that $G_{1} \cong G_{2}$. But by Theorems 1 and 3, we have just seen that sporadic simple groups and projective special linear simple group PSL $(2, p)$ uniquely determined by their main supergraph. So, we can pose the following question:

Question. Let $G$ be a finite group and $M$ a non-abelian finite simple group. Is it true that $G \cong M$ if only if $\mathcal{S}(G) \cong \mathcal{S}(M)$ ?

\section{Acknowledgment}

The authors would like to thank the referee for his/her careful reading and useful suggestions which led us to improve the paper.

\section{References}

[1] A. K. Asboei and A. Iranmanesh, A characterization of the linear groups $\operatorname{PSL}(2, p)$, Czechoslovak Math. J. 64(2) (2014), 459-464.

[2] A. K. Asboei, Characterizing $\operatorname{PSL}(2, r)$ by its order and the number of its Sylow r-subgroups, J. Algebra Appl. 13(1), 1350065 (2014).

[3] A. K. Asboei, Characterization of projective linear groups by the order of the normalizer of a Sylow subgroup, Monatsh Math. 173 (2014), 309-314.

[4] A. K. Asboei, A new characterization of PGL(2,p), J. Algebra Appl. 12(7), 1350040 (2013).

[5] A. K. Asboei and S. S. Salehi, Some alternating and symmetric groups and related graphs, Beitr Algebra Geom. 59 (2018), 21-24. 
[6] A. K. Asboei, S. S. Salehi, A. Iranmanesh A and A. Tehranian, A new characterization of sporadic simple groups by NSE and order, J. Algebra Appl. 12(2), 1250158 (2013).

[7] A. K. Asboei and S. S. Salehi, The small Ree group ${ }^{2} G_{2}\left(3^{2 n+1}\right)$ and related graph, Comment.Math.Univ.Carolin. 59(3), (2018), 271-276.

[8] A. K. Asboei and S. S. Salehi, Recognizability of finite groups by Suzuki group, Arch. Math. (Brno). 55 (2019), 225-228.

[9] P. J. Cameron, The power graph of a finite group, II, J. Group Theory. 13 (2010), 779-783.

[10] I. Chakrabarty, S. Ghosh and M. K. Sen, Undirected power graphs of semigroups, Semigroup Forum. 78 (2009), 410-426.

[11] J. H. Conway, R. T. Curtis, S. P. Norton, R. A. Parker and R. A. Wilson, Atlas of finite groups, Clarendon, Oxford, 1985.

[12] G. Frobenius, Verallgemeinerung des Sylow'schen Satzes, Berl. Ber. (1895), 981993. (In German)

[13] A. Hamzeh and A. R. Ashrafi, Automorphism groups of supergraphs of the power graph of a finite group, European J. Combin. 60 (2017), 82-88.

[14] B. Huppert, Endliche Gruppen, I, Springer, Berlin, 1967.

[15] A. Khosravi and B. Khosaravi, Two new characterization of sporadic simple groups, Pure Math. Appl. 16 (2005), 287-293.

[16] V. D. Mazurov and E. I. Khukhro, Unsolved problems in group theory, The Kourovka Notebook, (English version), ArXiv e-prints, (18), January 2014. Available at http://arxiv.org/abs/1401.0300v6.

[17] L. Zhang and X. Liu, Characterization of the projective general linear groups PGL $(2, q)$ by their orders and degree patterns, Int. J. Algebra Comput. 19 (2009), 873-889.

\section{CONTACT INFORMATION}

Alireza Khalili

Asboei

Seyed Sadegh

Salehi Amiri
Department of Mathematics, Farhangian

University, Tehran, Iran

E-Mail(s): a.khalili@cfu.ac.ir

Department of Mathematics, Babol Branch, Islamic Azad University, Babol, Iran

E-Mail(s): salehisss@baboliau.ac.ir

Received by the editors: 01.12 .2017

and in final form 18.03.2018. 